\title{
Caracterización de competencias profesionales necesarias para los médicos calificadores de la pérdida de la capacidad laboral y ocupacional en Colombia*
}

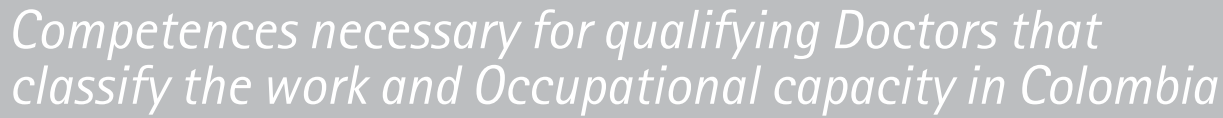

\author{
Hermes Rodolfo Suárez Vega** / Jorge Enrique Gallego Vásquez ${ }^{* * *}$ \\ / Paula Andrea Jaramillo Villegas*****
}

\section{RESUMEN}

El presente artículo es producto de la línea de investigación "Gestión educativa y curricular para la formación integral", desarrollada en la Maestría en Educación de la Corporación Universitaria Minuto de Dios. Esta identifica las competencias profesionales del médico para calificar la pérdida de capacidad laboral u ocupacional, que antes era llamada invalidez, en el marco colombiano de la Seguridad Social Integral. Este trabajo se basa en los saberes médicos, el marco legal de la calificación, y la revisión a cuatro planes posgraduales en Salud Ocupacional -ahora Seguridad y Salud en el Trabajo-. Asimismo, consulta a un grupo de médicos que integran las Juntas de Calificación de Invalidez, respecto a las competencias para calificar y necesidades de formación. A partir de los resultados, se puede apreciar que los programas hacen énfasis formativo en la prevención de la enfermedad laboral. No obstante, no desarrollan a profundidad la formación en calificación, especificamente en lo relacionado con la deficiencia / enfermedad. Por ello, se hace evidente la necesidad de crear planes de formación que incluyan competencias y saberes para el actuar médico en calificación, con responsabilidad social y ética, y que estas consideren el cambio de paradigmas sobre la calificación.

PALABRAS CLAVE: Deficiencia, evaluación médica independiente, ausencia por incapacidad, incapacidad laboral, invalidez, baremologia, daño corporal, deficiencia, pensión.

\begin{abstract}
This Article is the product of the research line "Educational and curricular management for integral training", developed in the Master's Degree in Education of the University Corporation Minuto de Dios. This identifies the professional competences of doctors to classify the loss of work or occupational capacity, which was previously called disability, with the Colombian framework of Integral Social Security, this work is based on Medical knowledge, the legal framework of qualifications, and the review of four postgraduate study plans in Occupational Healthnow called Health and Safety in the work place-. Nevertheless, it is consulted a group of doctors that make up the Disability Classification Boards, regarding prerequisite skills and training needs. From the results, it can be seen that the programs place a formative emphasis on the prevention of occupational disease. However, they do not develop indepth classification training, specifically in relation to the deficiency/disease. Therefore, it is evident the need to create training plans that include skills and knowledge to act in medical diagnosis, with social and ethical responsibility, and that these consider the paradigm shift on classification.
\end{abstract}

KEY WORDS: Deficiency, independent medical evaluation, absence due to disability, work disability, Disability, baremology, bodily harm, deficiency, pension.

* Artículo de investigación. Recibido el 5 de noviembre de 2018 y aceptado para su publicación el 1 de septiembre de 2019.

** Profesor emérito del Instituto de Posgrados de la Universidad Libre de Colombia. (hermessuarezv@ hotmail.com) orcid.org/000-0002-3754-6424 / *** Docente en la Corporación Universitaria Minuto de Dios, Colombia.

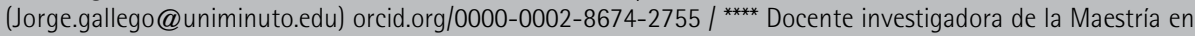
Educación e integrante del grupo geIEP de la Corporación Universitaria Minuto de Dios, Colombia. (pjaramillov@ uniminuto.edu.co) orcid.org/0000-0003-4580-3037 


\section{SUMARIO}

1. Introducción

2. Contexto de la calificación de la pérdida de capacidad laboral y ocupacional en Colombia: perspectiva investigativa

3. Calificación de la pérdida de capacidad laboral y ocupacional: perspectiva contextual

a) Competencias como marco general

b) Competencias en el contexto de la calificación de la pérdida de la capacidad laboral y ocupacional: perspectivas internacional y nacional

c) Enfoque metodológico

4. Conclusiones

\section{Introducción}

El marco de competencias en la formación del talento humano en materia de salud es hoy uno de los temas de mayor exigencia en el contexto colombiano. Este es fundamental para el ejercicio médico en lo que respecta a la valoración y calificación de las deficiencias generadas por una condición de salud. En el caso colombiano, estas acciones implican la aplicación de un baremo específico para determinar la pérdida de capacidad laboral y ocupacional, antes llamada calificación de invalidez.

Como fruto de la investigación -llevada a cabo en el marco de los requisitos para optar al título de magister en Educación de la Corporación Universitaria Minuto de Dios-, se presentan los principales hallazgos respecto a la caracterización de las competencias profesionales esperadas a la hora de valorar, calificar y determinar el valor porcentual de las deficiencias generadas por un evento profesional o común. Este determina para la población el acceso a derechos en materia de prestaciones económicas, como indemnizaciones y pensiones, según el Sistema de Seguridad Social colombiano. Calificar las deficiencias corresponde a aplicar el Título I: Valoración de las deficiencias, acorde a la norma colombiana; es este título específicamente el objeto de esta investigación.

\section{Contexto de la calificación de la pérdida de capacidad laboral y ocupacional en Colombia: perspectiva investigativa}

Según el marco legal colombiano, la calificación de la pérdida de capacidad laboral y ocupacional, antes llamada invalidez, históricamente ha demandado la presencia de profesionales en medicina en lo que respecta a la valoración de las deficiencias. Estas se entienden como aquellas condiciones clínicas articuladas 
a un diagnóstico médico que, desde la perspectiva actual de la Organización Mundial de la Salud, expresan problemas en las funciones y estructuras corporales en una persona. Para efectos de calificación se habla de secuelas, razón por la cual se hace necesaria la participación y articulación de equipos interdisciplinarios. En ellos, el rol médico se circunscribe a la calificación de las deficiencias, sin embargo, este aspecto aún es un reto colombiano, debido a la existencia de paradigmas descontextualizados, con los cuales se cree que la calificación es solo una práctica médica.

Para calificar es necesario el saber médico, puesto que su rol exige la consideración e incorporación de nuevas perspectivas y, por tanto, competencias especializadas. Estas, a su vez, deben tener soporte en el uso de herramientas técnicas que han de ser impartidas desde la academia. Todo ello evoca profundas reflexiones sobre las prácticas médicas, así como las necesidades y oportunidades que permitirian prestar un mejor servicio. En especial a personas que, por cualquier contingencia, son sujetos de derecho a dictámenes o calificaciones periciales, las cuales deben estar basadas en saberes, competencias, prácticas y conceptos asertivos, equitativos, éticos y de calidad, a partir de la formación académica y profesional.

Las investigaciones sobre el tema resultan escasas, razón por la cual el problema de investigación partió del saber propio del investigador, la experiencia laboral y docente acumulada por más de 25 años. El objetivo fue caracterizar las competencias profesionales de los médicos calificadores a partir del análisis a los programas académicos de especialización médica que establece el marco legal en Colombia para la calificación del Título I, Valoración de las deficiencias del Decreto 1507 de 2014. Los objetivos específicos se orientan a analizar los elementos conceptuales sobre la calificación de las deficiencias o Título I del Decreto en los planes de estudio de la especialización de Salud Ocupacional (hoy Seguridad y Salud en el trabajo) y en Medicina del Trabajo, de la ciudad de Bogotá, Barranquilla, Cali y Medellín. Asimismo, se buscó registrar las necesidades de formación y gestión del conocimiento que considera un grupo de médicos calificadores integrantes de las Juntas de Calificación de Invalidez como relevantes para la mejora de las competencias profesionales requeridas a la hora de calificar las deficiencias, y sugerir un conjunto de competencias profesionales necesarias que debe tener el médico calificador a la hora de determinar el grado de severidad de las deficiencias.

Si bien el tema de investigación podría ser más ambicioso, se encontraron límites temporales y espaciales, así como a problemáticas que hoy en día representan conflictos éticos y morales en Colombia. A esto se suma que, si bien el estudio podría ser más potente al haber analizado currículos, hubo 
restricciones de acceso a los mismos y, por tanto, no fueron parte de la definición operacional del investigador. Por ello, precisamente, la investigación en sí misma buscó responder a qué dicen los planes de estudios de los programas de posgrado respecto a las competencias orientadas al tema de calificación de pérdida de capacidad laboral y ocupacional, correlacionada con los objetivos anteriormente expuestos.

La metodología fue de enfoque mixto, pues, de acuerdo con Hernández, Fernández y Baptista, ${ }^{1}$ entre otras ventajas, esta logra una perspectiva más amplia y profunda para caracterizar las competencias de los médicos calificadores. Respecto a la población objeto, según Lepkowski, ${ }^{2}$ es "el conjunto de todos los casos que concuerdan con una serie de especificaciones”. La investigación tuvo en cuenta dos grupos.

El primer grupo fue el de los médicos integrantes de las Juntas de Calificación de Invalidez, se seleccionó por muestreo no probabilístico o dirigido por conveniencia (también conocido como "guiadas por uno o varios propósitos"). Este fue indagado mediante una encuesta, teniendo en cuenta la elección de los elementos que dependen de las razones relacionadas con las características propias de la investigación. ${ }^{3}$

El segundo grupo hace referencia a los programas de posgrado en la especialización en Salud Ocupacional o Medicina del Trabajo que ofrecen las entidades educativas. Estos programas académicos son el único requisito en términos de formación posgradual requerido para ser miembro de las Juntas de Calificación de Invalidez. La muestra de estos programas se ubicó en las ciudades sede de las Juntas de Calificación, seleccionadas del primer grupo de estudio, a cuyos integrantes se les aplicó la técnica de análisis cualitativo, desde la perspectiva general de contenido. Cabe resaltar que un plan de estudios no permite profundizar en los currículos o mallas curriculares; sin embargo, la información arrojada resulta valiosa y permite ubicar desde un análisis de contenido la situación de la formación académica en Colombia.

1 Hernández Sampieri, Roberto, Fernández, Carlos y Baptista, Pllar, "Metodologías de la Investigación", México, Mc Graw Hill, 2014, p. 570.

2 Hernández, Roberto, Fernández, Carlos y Baptista, Pilar, "Metodologías de la Investigación", México, Mc Graw Hill, 2014, p. 174.

3 Hernández, Roberto, Fernández, Carlos y Baptista, Pllar, "Metodologías de la Investigación", México, Mc Graw Hill, 2014, p. 386. 


\section{Calificación de la pérdida de capacidad laboral y ocupacional: perspectiva contextual}

Hablar de la calificación de pérdida de capacidad laboral y ocupacional implica ubicarse históricamente a mediados del siglo xx, donde el médico calificador de la "invalidez" partía de lo anátomo funcional. Así, esta se determinaba con base en tablas de valuación del daño que establecían los valores mínimos y máximos, según la edad del trabajador y la incidencia con el ejercicio de la profesión o trabajo remunerado. De este modo, no se tenían en cuenta los aspectos socio económicos y la incidencia sobre las actividades de la vida diaria que presentaba la persona como consecuencia de la enfermedad.

A partir de 1994, entrado el nuevo Sistema de Seguridad Social Integral en Colombia, la determinación de la calificación de la invalidez inició con el criterio de establecer el grado de severidad de la deficiencia, generado por la enfermedad o el accidente. Asimismo, se sumaron las consecuencias que presenta el calificado en el funcionamiento dentro del contexto socioeconómico, laboral y en la ejecución de las actividades de la vida diaria. En otras palabras, se comenzaron a considerar los efectos propios de las personas enfermas, como son las limitaciones de la actividad de la vida diaria y las desventajas de orden social, económico, ocupacional, en función de la edad, para determinar la, en aquel entonces, discapacidad o minusvalía. De este modo, lo anterior se pudo expresar como pérdida de un grado o más en la capacidad laboral, hasta llegar a la invalidez.

Con base en los avances científicos, a partir del año 2009, Colombia inició la actualización y modificación de la herramienta para la calificación o baremo concebido para tal fin. Al respecto, es importante tener en cuenta que esta herramienta y las anteriores, adoptadas por decretos reglamentarios, son normas técnicas basadas en estándares internacionales que actualmente no están vigentes; por ejemplo: El Manual Diagnóstico y Estadístico de los Trastornos Mentales IV (en inglés, Diagnostic and Statistical Manual of Mental Disorders, abreviado dsm IV), la Clasificación Internacional de Enfermedades $10 .^{\text {a revisión }}$ (CIE 10), clasificación vigente en Colombia y la Clasificación Internacional de las Deficiencias, Discapacidades y Minusvalías (CIDDM) ahora CIF. ${ }^{4}$

En este orden de ideas, desde el año 1999 hasta el 2014, Colombia hablaba de calificar las deficiencias, discapacidades y minusvalías. La norma actual de calificación -motivo del presente estudio- fue adoptada por el Decreto 1507

\footnotetext{
${ }^{4}$ Organización Mundial de la Salud, Clasificación Internacional del Funcionamiento, la Discapacidad y la Salud, Ginebra, Organización Mundial de la Salud, 2001.
} 
de 2014. ${ }^{5}$ Como lo relata Moreno, esto fue resultado de un proceso arduo de construcción acompañado de espacios de debate y reflexión participativa que vinculó a diferentes sectores del campo de la Seguridad Social en Colombia, ${ }^{6}$ desde el sector público y privado hasta el sector académico. Asimismo, cabe resaltar la importancia y el avance de Colombia, al haber incorporado la Clasificación Internacional de Funcionamiento, de la Discapacidad y de la Salud (CIF) de la Organización Mundial de la Salud; ${ }^{7}$ esto, acompañado de espacios de encuentro para reflexionar sobre el campo metodológico y conceptual de manuales y baremos de calificación a nivel nacional e internacional.

El decreto contiene dos partes denominadas títulos. ${ }^{8}$ El primero, llamado valoración de las deficiencias, se relaciona con la condición de salud de las personas, específicamente a nivel de secuelas por eventos de cualquier origen y que afectan las funciones o estructuras corporales; es decir donde el actuar médico es necesario. El segundo, denominado valoración del rol laboral, rol ocupacional y otras áreas, se deja abierto a diferentes disciplinas (propias de los terapeutas y psicólogos). Para el investigador tal aspecto ya resulta confuso, dado que las competencias y saberes entre estos títulos son diferentes y allí es donde se generan grandes debates y reflexiones. Se hace necesario diferenciar el rol del calificador, respecto a roles importantes como los interconsultores. Cabe aclarar que este segundo título no es objeto de esta investigación.

Con base en lo anterior, la Ley 100 de 1993 y las normas que la complementan (como la Ley 776 de 2002, la Ley 797 de 2013 y la Ley 860 de 2003) establecen los requisitos para obtener las pensiones de invalidez de origen común o profesional, de la de pensión de vejez anticipada por hijos inválidos a cualquier edad y por deficiencias iguales o mayores a 50\%, como lo regula taxativamente la norma general, diferente al estado de invalidez. Uno de los requisitos es que la persona presente una incapacidad permanente parcial o una invalidez, determinada con el Manual Único de Calificación para la Pérdida de la Capacidad Laboral y Ocupacional.

Para lo anterior, se define que las Juntas de Calificación de Invalidez, como organismos privados vigilados por el Ministerio de Trabajo y Protección Social, cuentan con la función de expedir dictámenes en primera y segunda

\footnotetext{
${ }^{5}$ Adoptado por el Decreto 1507 de 2014 por el cual se expide el Manual Único para la Calificación de la Pérdida de la Capacidad Laboral y Ocupacional.

${ }^{6}$ Moreno, MarisoL, Valoración de la pérdida de la capacidad laboral y ocupacional en Colombia: antecedentes, realidades y perspectivas de actualización, Bogotá, Universidad Nacional de Colombia, 2011, pp. 234-265.

7 Organización Mundial de LA Salud, Clasificación Internacional del Funcionamiento, la Discapacidad y la Salud, Ginebra, Organización Mundial de la Salud, 2001.

${ }^{8}$ Uno de los referentes en la construcción del baremo colombiano partió de la Organización Mundial de LA SALUd, Clasificación Internacional del Funcionamiento, la Discapacidad y la Salud, Ginebra, Organización Mundial de la Salud, 2001.
} 
instancia. ${ }^{9}$ Esto puede asimilarse como prácticas médicas que, en el marco de juntas, aportan pruebas de tipo pericial.

Ahora bien, en materia de requisitos, para ser miembro médico de estas juntas, es preciso contar con título de médico y formación posgradual en salud ocupacional o medicina del trabajo. No obstante, si bien se establecen estas exigencias para la profesión médica en el campo de la calificación, se cuenta con una pobre evidencia de estudios sobre las competencias profesionales para los médicos calificadores, los cuales serían requisitos necesarios para la experticia que demanda el acto de valorar y calificar. Las indagaciones llevadas a cabo denotan que, solo desde el 2014, algunos médicos calificadores iniciaron procesos de capacitación bajo modalidades de educación informal, dado el nuevo baremo desarrollado en Colombia.

La intención de esta investigación se hace más relevante si se tiene en cuenta que se ha de contar con un perfil de competencias profesionales frente a un actuar que denota el acceso a derechos en el marco del Sistema de Seguridad Social Integral (sssi) en Colombia. Teniendo en cuenta esta situación, se presume que existe un problema actual, sumado a escasos estudios respecto a la formación de los profesionales médicos en el tema objeto de este trabajo.

De acuerdo con Sosa y Capote, ${ }^{10}$ el estudio de la evaluación de las competencias ocupa hoy un espacio de primer orden y es abordado por diversas instituciones y autores. Las competencias profesionales no constituyen características que se adquieren de una vez y para siempre; más bien, son procesos que incrementan las potencialidades a partir de avances secuenciales. En el actuar médico, estos cobran mayor relevancia cuando está de por medio la definición de derechos económicos en el marco del Sistema de Seguridad Social Integral en Colombia.

$\mathrm{Al}$ respecto, la determinación de las competencias en el marco de la calificación de la pérdida de capacidad laboral y ocupacional permitirá caracterizar las competencias profesionales necesarias que deben tener los médicos laborales. Esto no solo para una mejor prestación de los servicios, sino en especial para generar profundas reflexiones en torno a la situación actual de la formación posgradual relacionada con el tema en cuestión. Asimismo, tales competencias son un tema relevante por el impacto que pueden tener, en especial para las personas que día a día acuden a estos procesos y a las instituciones que

\footnotetext{
${ }^{9}$ Las Juntas de Calificación de Invalidez, creadas por el artículo 42 y 43 de la Ley 100 de 1993 y reglamentadas por el decreto 1352 de 2013.

${ }^{10}$ Sosa, Ángela y Capote, Silvia, "Modelo tradicional versus enfoque por competencias", Uruguay Educa. Disponible en: http://www.uruguayeduca.edu.uy/sites/default/files/2017-08/MODELO_TRADICIONAL_vs_ENFOQUE_por_COMPETENCIAS.pdf
} 
contratan y delegan en los médicos la evaluación y determinación del grado porcentual de pérdida de la funcionalidad de las estructuras corporales, solo para el título de deficiencias.

Hasta donde indica la evidencia, los médicos actualmente cuentan con formación de pregrado en Medicina General y, en algunos casos, formación posgraduada en Salud Ocupacional (ahora Salud y Seguridad en el Trabajo). Con estos requisitos, pueden ser calificadores sin parámetros definidos en materia de competencias para calificar, más aún cuando cualquier persona, de cualquier edad, con eventos por origen laboral o común, son sujetos de calificación. Ello significa que el rol de estos profesionales no puede sesgarse solo al campo de lo laboral o de personas trabajadoras. En palabras de Perrenoud, ${ }^{11}$ "la profesión no es inmutable. Sus transformaciones pasan sobre todo por la aparición de nuevas competencias [...] o por el énfasis de competencias reconocidas”. Es así como el desempeño profesional en un terreno específico exige actualizaciones permanentes para comprender la práctica profesional y, de paso, mejorar constantemente la experticia de la calificación. Esto cobra aún más relevancia cuando a las personas no se les puede ver solo desde un modelo biomédico y más allá de un diagnóstico.

La gestión del conocimiento, la incorporación de las tecnologías; los avances en la medicina de hoy, así como los requerimientos actuales de la seguridad social exigen nuevos roles que respondan a los cambios. Todo ello hace necesario pensar en los nuevos conocimientos, habilidades y actitudes que debe tener un médico, en este caso calificador. Su énfasis consiste en valorar secuelas de condiciones de salud relacionadas con contingencias o eventos denominados enfermedad o accidente, sin importar su origen. Ante todo, pesa el acceso a derechos y el reconocimiento de que mucha población colombiana no trabaja, no ha trabajado y en el marco de pensiones entran como beneficiarios. Aquí se marca un actuar diferente, porque se debe reconocer el rol interdisciplinar y los aportes de la Clasificación Internacional del Funcionamiento, la Discapacidad y la Salud (CIF) en la comprensión actual del funcionamiento, la discapacidad y, para el caso colombiano, lo que significa la invalidez, más allá de un valor numérico.

\footnotetext{
"Diaz, Martha, "Reseña de Diez nuevas competencias para enseñar de Philippe Perrenoud", Tiempo de Educar, vol. 9, núm. 17. Disponible en: http://ucsj.redalyc.org/articulo.oa?id=31111439008
} 
Ante estos argumentos, la pertinencia de investigar, documentar la realidad y proponer cambios en torno a las competencias necesarias para la formación integral de un profesional en el contexto de la calificación, se busca ahondar en la gestión del conocimiento. Así, cabe repensar y reconstruir el perfil de los médicos calificadores a partir de un análisis no solo desde sus saberes, experiencias y vivencias frente a la calificación misma, sino desde el entorno académico.

Para tal efecto, el estudio revisó cuatro planes de estudio posgraduales relacionados con los programas en Salud Ocupacional y Medicina del Trabajo, ofrecidos en las ciudades de Bogotá, Barranquilla, Cali y Medellín. Para ello, se analizaron los elementos conceptuales que constituyen los módulos o materias sobre la calificación de las deficiencias ${ }^{12}$ o el daño corporal.${ }^{13}$ Relacionado con lo anterior, se identificó la percepción de los médicos miembros de las Juntas de Calificación, respecto a las necesidades de formación y gestión del conocimiento, para la mejora de las competencias profesionales requeridas a la hora de calificar.

\section{a) Competencias como marco general}

De acuerdo con Dao, ${ }^{14}$ la Organización Panamericana de la Salud (ops), a mediados de la década de los ochenta, comenzó a promover entre los países de la región de las Américas la propuesta de reorientación de la educación permanente del personal de salud. Es por ello que, al momento de evaluar las competencias laborales de los médicos califıcadores y el desempeño en el actuar profesional, apuestas como las de la oms pueden ser extrapoladas al contexto de la calificación. Esto se complementa con diferentes perspectivas que se exponen en la tabla 1.

\footnotetext{
${ }^{12} \mathrm{El}$ artículo 3 del Decreto 1507 de 2014 define la deficiencia como la "Alteración en las funciones fisiológicas o en las estructuras corporales de una persona. Puede consistir en una pérdida, defecto, anomalía o cualquier otra desviación significativa respecto de la norma estadísticamente establecida".

${ }^{13}$ El artículo 3 del Decreto 1507 de 2014 define el daño corporal: "Concepto que resulta de la confluencia de dos perspectivas, la médica y la jurídica. Con el nombre de daño corporal se conoce cualquier alteración somática o psíquica que, de una forma $u$ otra, perturbe, amenace o inquiete la salud de quien la sufre, o simplemente, limite o menoscabe la integridad personal del afectado, ya en lo orgánico, ya en lo funcional; para que se configure, es suficiente cualquier merma de la integridad de la biologia individual, con independencia de sus repercusiones prácticas en uno o más campos de la actividad humana".

${ }^{14}$ DAO, AlberTo, El conocimiento y las competencias en las organizaciones del siglo XXI, Venezuela, Epsilon Libros Universidad Metropolitana, 2002.
} 


\section{Tabla 1. Perspectivas y evolución del concepto de competencias}

\begin{tabular}{|c|c|}
\hline Autor y año & Definición o aporte al concepto de competencia \\
\hline $\begin{array}{r}\text { Noam } \\
\text { Chomsky } \\
(1957)\end{array}$ & $\begin{array}{l}\text { Propuso la competencia desde la lingüistica en 1957, como la capacidad que tiene todo ser } \\
\text { humano de manera innata de hablar y crear mensajes que nunca habia oído. }\end{array}$ \\
\hline $\begin{array}{r}\text { Dell Hymes } \\
(1972)\end{array}$ & $\begin{array}{l}\text { Aportó el concepto de competencia comunicativa como aquella que permite a un hablante } \\
\text { comunicarse de manera eficaz en contextos culturalmente significantes. Es integral con } \\
\text { actitudes, valores y motivaciones. }\end{array}$ \\
\hline $\begin{array}{r}\text { Gerhard Bunk } \\
(1994)\end{array}$ & $\begin{array}{l}\text { Tiene competencia profesional quien dispone de conocimientos, destrezas y aptitudes nece- } \\
\text { sarios para ejercer una profesión, resuelve los problemas profesionales de forma autónoma } \\
\text { y flexible, y está capacitado para colaborar en su entorno profesional. }\end{array}$ \\
\hline $\begin{array}{r}\text { Fabio Jurado } \\
(2000)\end{array}$ & $\begin{array}{l}\text { La competencia es un saber hacer que el sujeto porta en determinado campo, y que solo } \\
\text { es posible identificar en la acción misma; le ayuda al sujeto a desenvolverse en la vida } \\
\text { práctica y a construir un horizonte social. }\end{array}$ \\
\hline $\begin{array}{r}\text { Guy Le Boterf } \\
(2001)\end{array}$ & $\begin{array}{l}\text { La competencia es un proceso, "una secuencia de acción en la que se encadenan múltiples } \\
\text { conocimientos especializados" (p. 42). Implica saber integrar, movilizar y transferir un } \\
\text { conjunto de recursos en un contexto dado para hacer frente a diferentes problemas que } \\
\text { se presentan. }\end{array}$ \\
\hline $\begin{array}{r}\text { María Cristina } \\
\text { Torrado } \\
(2000) \\
\end{array}$ & $\begin{array}{l}\text { Las competencias son capacidades individuales que son condición necesaria para impulsar } \\
\text { un desarrollo social en términos de equidad y ejercicio de la ciudadanía. }\end{array}$ \\
\hline $\begin{array}{r}\text { Carlos A. } \\
\text { Brailovsky } \\
(2001)\end{array}$ & $\begin{array}{l}\text { "La competencia es un constructo complejo, multifacético, multivariado, multidimensional, } \\
\text { a menudo en relación con una situación multidisciplinaria, en particular en el campo de } \\
\text { la medicina" (p.103). }\end{array}$ \\
\hline $\begin{array}{r}\text { María Luisa } \\
\text { Belloto e } \\
\text { Imma Palma } \\
\text { Linares } \\
(2008)\end{array}$ & $\begin{array}{l}\text { Un sistema de conocimientos, habilidades, valores y cualidades de la personalidad que se } \\
\text { movilizan en función de las necesidades individuales y sociales, así como de los motivos, } \\
\text { intereses y actitudes del profesional, que permiten el desempeño satisfactorio en el ejercicio } \\
\text { de la profesión; y que solo pueden ser evaluadas a través del desempeño, considerando } \\
\text { las exigencias sociales. }\end{array}$ \\
\hline $\begin{array}{r}\text { Philppe } \\
\text { Perrenoud } \\
(2010 a)\end{array}$ & $\begin{array}{l}\text { La capacidad de actuar de manera eficaz en un tipo definido de situación; se apoya en } \\
\text { conocimientos, pero no se reduce a ellos. Se enfrenta una situación de la mejor manera } \\
\text { posible, generalmente debemos hacer uso y asociar varios recursos cognitivos complemen- } \\
\text { tarios, entre los cuales se encuentran los conocimientos (p.7). }\end{array}$ \\
\hline $\begin{array}{r}\text { Polform/ } \\
\text { OIT Ducci, M. } \\
(1997)\end{array}$ & $\begin{array}{l}\text { La competencia profesional es vista como la construcción social de aprendizajes signi- } \\
\text { ficativos y útiles para el desempeño productivo en una situación real de trabajo que se } \\
\text { obtiene no solo a través de la instrucción, sino también -y en gran medida- mediante } \\
\text { el aprendizaje por experiencia en situaciones concretas de trabajo. Para la olt, parte de } \\
\text { la idoneidad para realizar una tarea o desempeñar un puesto de trabajo por poseer las } \\
\text { calificaciones requeridas para ello. }\end{array}$ \\
\hline $\begin{array}{r}\text { Australia- } \\
\text { Athanasou } \\
\text { y Gonczi } \\
(1996)\end{array}$ & $\begin{array}{l}\text { Conciben la competencia como una compleja estructura de atributos (conocimiento, acti- } \\
\text { tudes, valores y habilidades) necesarios para el desempeño de situaciones especificas y las } \\
\text { tareas que se tienen que desempeñar en determinadas situaciones. Encuentran necesario } \\
\text { incorporar la ética y los valores como elementos del desempeño competente. }\end{array}$ \\
\hline
\end{tabular}

Nota: Tomado de Moreno, L. (2015). Adaptada por Suarez, H. (2018). 
b) Competencias en el contexto de la calificación de la pérdida de la capacidad laboral y ocupacional: perspectivas internacional y nacional

Como lo expone Moreno y Rubio, ${ }^{15}$ la evolución y aplicación de los baremos en el contexto internacional demanda actualmente la inclusión de profesionales competentes para calificar o evaluar según las épocas, el tipo de legislación, los hechos resaltables y los principios de reparación del daño, a partir de lo puramente médico (hechos relacionados en la tabla 2). Por lo anterior, y como lo registra Kvitko, ${ }^{16}$ la evolución histórica contempla la generación e identificación de competencias relacionadas con la valoración del daño. Ejemplo de ello fue el primer baremo español sobre las incapacidades originadas por los accidentes de trabajo. Hoy, esto resulta válido para el baremo colombiano, al valorarse las deficiencias generadas por una enfermedad o accidente.

Tabla 2. Síntesis de la evolución de los baremos en el mundo

\begin{tabular}{|l|l|l|l|}
\hline \multicolumn{1}{|c|}{ Época } & \multicolumn{1}{|c|}{ Tipo de legislación } & \multicolumn{1}{|c|}{ Hecho resaltable } & \multicolumn{1}{|c|}{$\begin{array}{c}\text { Principio de } \\
\text { evaluación }\end{array}$} \\
\hline Edad Media & $\begin{array}{l}\text { Ley Sálica del siglo } \mathrm{V} \\
\text { Francia siglos XI y xII } \\
\text { Leyes Normandas }\end{array}$ & $\begin{array}{l}\text { Se establece la } \\
\text { necesidad de un } \\
\text { perito }\end{array}$ & $\begin{array}{l}\text { Médico competente } \\
\text { para examinar las } \\
\text { heridas }\end{array}$ \\
\hline Renacimiento & $\begin{array}{l}\text { Constitutio Criminalis } \\
\text { Carolina (1532) }\end{array}$ & $\begin{array}{l}\text { Médico en actos } \\
\text { jurídicos }\end{array}$ & $\begin{array}{l}\text { Constitución de la } \\
\text { medicina legal }\end{array}$ \\
\hline Barroco (siglo xvI) & La obra de Zacchia & $\begin{array}{l}\text { Primer tratado de } \\
\text { medicina legal }\end{array}$ & $\begin{array}{l}\text { De competencia } \\
\text { médica }\end{array}$ \\
\hline $\begin{array}{l}\text { Positivismo } \\
\text { naturalista (siglo xIX) }\end{array}$ & $\begin{array}{l}\text { España crea el primer } \\
\text { cuerpo nacional de } \\
\text { médicos forenses }\end{array}$ & $\begin{array}{l}\text { De competencia } \\
\text { médica }\end{array}$ \\
\hline $\begin{array}{l}\text { Finales del siglo xIx, } \\
\text { Francia }\end{array}$ & Baremos militares & $\begin{array}{l}\text { Un grupo de médicos } \\
\text { integra al baremo } \\
\text { terminología médica, } \\
\text { dando un orden } \\
\text { lógico a las secuelas. }\end{array}$ & \\
\hline
\end{tabular}

Nota: Tomado del cuadro 3,9 expuesto por Moreno y Rubio (2011) y adaptada por Suarez (2018): Síntesis de la evolución de los baremos en el mundo.

\footnotetext{
${ }^{15}$ Moreno, Marisol, Valoración de la pérdida de la capacidad laboral y ocupacional en Colombia: antecedentes, realidades y perspectivas de actualización, Bogotá, Universidad Nacional de Colombia, 2011.

${ }^{16}$ KVITKo, LuIS, "Antecedentes Históricos de la Valoración del Daño Corporal y Baremos", Asociación Costarricense de Medicina Legal, vol. 32, núm. 81. Disponible en: http://www.scielo.sa.cr/pdf/mlcr/v32n1/art08v32n1.pdf
} 
En el contexto colombiano, ha habido diferentes normas, circunscritas a distintos momentos históricos y perspectivas tales como el Acuerdo 258 de 1967 del Instituto de Seguros Sociales; Decreto 692 de 1995; Decreto 917 de 1999, y el más reciente y vigente, Decreto 1507 de 2014. Todas estas denotan una evolución, sobre todo a nivel legal. La tabla 3 ilustra los aspectos más relevantes, no solo respecto a los baremos, sino a los diferentes referentes o normas que se interconectan con la calificación. Es claro cómo el rol del médico se ha mantenido; no obstante, el acto de calificar sí se ve expuesto a diferentes avances en el conocimiento y cambios de paradigma que no necesariamente se tienen en cuenta en la norma como tal.

\section{Tabla 3. Evolución de la calificación en Colombia según marco legal}

\begin{tabular}{|c|c|c|}
\hline Norma & Características & Relación con el marco de la calificación \\
\hline $\begin{array}{l}\text { Código } \\
\text { Sustantivo } \\
\text { de Trabajo y } \\
\text { Decreto } 3170 \\
\text { de } 1964^{2}\end{array}$ & \multirow[t]{2}{*}{$\begin{array}{l}\text { Establece el Seguro } \\
\text { Social obligatorio } \\
\text { por accidentes de } \\
\text { trabajo y enfermedades } \\
\text { profesionales, asumido } \\
\text { y administrado por el } \\
\text { Instituto Colombiano } \\
\text { de Seguros Sociales. }\end{array}$} & $\begin{array}{l}\text { Da inicio al reconocimiento de prestaciones } \\
\text { económicas. Considera los grados de } \\
\text { incapacidad permanente, parcial o total, } \\
\text { que presente un enfermo o un accidentado } \\
\text { laboral. Se apoya en las tablas de evaluaciones } \\
\text { de incapacidades, a cargo del Departamento } \\
\text { Médico Legal del lnstituto, las cuales } \\
\text { determinaban los grados de incapacidad. }\end{array}$ \\
\hline $\begin{array}{l}\text { Acuerdo } 049 \\
\text { de febrero } 1 \text { de } \\
1990^{3} \text { y Decreto } \\
758 \text { de } 1990^{4}\end{array}$ & & $\begin{array}{l}\text { Describe que, para efectos de la calificación } \\
\text { del grado de invalidez, solo son válidas las } \\
\text { efectuadas por los médicos laborales del } \\
\text { Instituto Colombiano de Seguros Sociales. }\end{array}$ \\
\hline Ley 100 de $1993^{5}$ & $\begin{array}{l}\text { Crea el marco del } \\
\text { sssi dado por los } \\
\text { regímenes en Salud, } \\
\text { Riesgos Laborales y en } \\
\text { Pensiones. Establece } \\
\text { las prestaciones de } \\
\text { carácter económico. }\end{array}$ & $\begin{array}{l}\text { La calificación del estado de invalidez se } \\
\text { determina con el manual único, para calificar } \\
\text { la imposibilidad que tenga el afectado para } \\
\text { desempeñar su trabajo por pérdida de la } \\
\text { capacidad laboral. La calificación es dada } \\
\text { por una comisión interdisciplinaria. }\end{array}$ \\
\hline $\begin{array}{l}\text { Decreto } 1346 \\
\text { de } 1994^{6}\end{array}$ & $\begin{array}{l}\text { Norma reglamentaria } \\
\text { de las Juntas de } \\
\text { Calificación de Invalidez } \\
\text { vigencia de la } \\
\text { Ley } 100 \text { de } 1993 .\end{array}$ & $\begin{array}{l}\text { Establece la calificación de los estados de } \\
\text { invalidez por las administradoras del sssı y, } \\
\text { en el caso de controversia o apelación, son las } \\
\text { juntas regionales y nacional de calificación } \\
\text { de invalidez las que califican, respectivamente. } \\
\text { Estas juntas se conforman por dos médicos } \\
\text { con título de especialización en medicina } \\
\text { del trabajo, o salud ocupacional y un psicólogo. } \\
\text { Los ponentes son los médicos. }\end{array}$ \\
\hline
\end{tabular}




\begin{tabular}{|c|c|c|}
\hline $\begin{array}{l}\text { Decreto } 2463 \\
\text { de } 2001^{7}\end{array}$ & $\begin{array}{l}\text { Mantiene la } \\
\text { interdisciplinaridad } \\
\text { de las Juntas de } \\
\text { Calificación de Invalidez, } \\
\text { dadas en el decreto } \\
\text { antes mencionado. }\end{array}$ & $\begin{array}{l}\text { Las entidades administradoras de la } \\
\text { seguridad social deben contar con equipo } \\
\text { interdisciplinario para realizar la calificación } \\
\text { de la pérdida de la capacidad laboral. El equipo } \\
\text { debe estar conformado por un médico con } \\
\text { experiencia en medicina laboral, un médico } \\
\text { especialista en medicina física y rehabilitación } \\
\text { y un profesional diferente a las áreas de la } \\
\text { medicina con formación en salud ocupacional. }\end{array}$ \\
\hline $\begin{array}{l}\text { Decreto ley } 1295 \\
\text { de } 1994^{8}\end{array}$ & $\begin{array}{l}\text { Establece las } \\
\text { prestaciones } \\
\text { económicas } \\
\text { (indemnización por } \\
\text { incapacidad permanente } \\
\text { parcial y la pensión } \\
\text { por invalidez). }\end{array}$ & $\begin{array}{l}\text { La declaración, evaluación, revisión, } \\
\text { grado y origen de la incapacidad permanente } \\
\text { parcial son determinados por un médico o por } \\
\text { una comisión médica interdisciplinaria. }\end{array}$ \\
\hline $\begin{array}{l}\text { Decreto Ley } 962 \\
\text { de } 2005^{9}\end{array}$ & $\begin{array}{l}\text { Modifica el artículo } 41 \\
\text { de la Ley } 100 \text { (1993) } \\
\text { con el artículo } 52 .\end{array}$ & $\begin{array}{l}\text { Mantiene la competencia a las administradoras } \\
\text { del sssi y, en el caso de controversia o } \\
\text { apelación, son las juntas regionales y nacional } \\
\text { de calificación de invalidez las que califican, } \\
\text { respectivamente. Estas juntas se conforman } \\
\text { por dos médicos con título de especialización } \\
\text { en Medicina del Trabajo, o salud ocupacional } \\
\text { y un psicólogo. }\end{array}$ \\
\hline $\begin{array}{l}\text { Ley } 776 \\
\text { de } 2002^{10}\end{array}$ & $\begin{array}{l}\text { Esta ley se expide } \\
\text { en respuesta al } \\
\text { fallo emitido por } \\
\text { la Honorable Corte } \\
\text { Constitucional al } \\
\text { declarar parcialmente } \\
\text { inexequible el Decreto } \\
1295 \text { (1994). }\end{array}$ & $\begin{array}{l}\text { La declaración, evaluación, revisión, } \\
\text { grado y origen de la incapacidad permanente } \\
\text { parcial los determina una comisión médica } \\
\text { interdisciplinaria. }\end{array}$ \\
\hline $\begin{array}{l}\text { El Decreto Ley } \\
019 \text { de } 2012^{11}\end{array}$ & $\begin{array}{l}\text { Faculta nuevamente y } \\
\text { con mayor fuerza legal } \\
\text { las competencias para } \\
\text { determinar la pérdida } \\
\text { de la capacidad laboral, } \\
\text { la invalidez y el origen } \\
\text { de las contingencias. }\end{array}$ & $\begin{array}{l}\text { Mantiene la competencia a las administradoras } \\
\text { del sssl, de determinar en primera oportunidad } \\
\text { la pérdida de capacidad laboral y calificar el } \\
\text { grado de invalidez y establece que las Juntas } \\
\text { de Calificación de lnvalidez del orden regional } \\
\text { califican en primera y segunda instancia, } \\
\text { respectivamente. }\end{array}$ \\
\hline $\begin{array}{l}\text { Decreto } 1352 \\
\text { de } 2013^{12}\end{array}$ & $\begin{array}{l}\text { Reglamenta la } \\
\text { organización y } \\
\text { funcionamiento de las } \\
\text { Juntas de Calificación } \\
\text { de Invalidez }\end{array}$ & $\begin{array}{l}\text { La Junta Nacional de Calificación de lnvalidez } \\
\text { la conforma tres médicos con título de } \\
\text { especialización en salud ocupacional o } \\
\text { medicina del trabajo y un médico fisiatra. Las } \\
\text { Juntas Regionales de Calificación de Invalidez, } \\
\text { las conforma dos médicos con especialización } \\
\text { en medicina del trabajo o salud ocupacional y } \\
\text { un psicólogo o terapeuta físico u ocupacional. }\end{array}$ \\
\hline
\end{tabular}

Nota: Tabla elaborada por Rodríguez, F. y Suárez, H. (2018). 
Con base en lo expuesto, es preciso identificar y reflexionar en torno al tema de las competencias médicas relacionadas con la valoración de los daños causados por las enfermedades y los accidentes que presentan las personas, en términos actuales y correctos; es decir, lo que hoy significa valorar las deficiencias. En este orden de ideas, la diversificación de los conocimientos médicos ha dado lugar a la determinación de campos de profundización en la práctica médica mediante programas de educación posgradual o continua. Se espera que estos sean pertinentes en torno a un marco de competencias para correlacionar el actuar, actividad, servicio, deber ser, objetivo y construcción desde la misma academia.

Ahora bien, el Decreto 1507 de 2014, ${ }^{17}$ denominado Manual Único para la Calificación de la Pérdida de la Capacidad Laboral y Ocupacional, establece una estructura conformada por dos títulos. El título 1, "Valoración de las deficiencias", está conformado por 15 capítulos (véase la tabla 4) que se refieren al estudio desde la perspectiva de secuelas. Esto es qué pasa en los órganos, estructuras o funciones que componen el cuerpo humano, y el médico calificador asigna valores porcentuales, dados en las tablas específicas, según clasificación del grado de severidad de cada deficiencia. Estos valores van desde 0\% hasta 50\% ponderado y definen el impacto a nivel de funcionalidad.

Sumado a lo anterior, se da lo dispuesto desde el título 2: "Valoración del rol laboral, ocupacional y otras áreas ocupacionales" (actividades de la vida diaria-AVD). Este permite obtener otro valor porcentual comprendido entre $0 \%$ y 50\%. Es decir, una vez sumados los dos títulos, resulta el valor total que desde $0 \%$ a 100\% define cuantitativamente la pérdida de la capacidad laboral $\mathrm{u}$ ocupacional para la población en cualquier edad, ocupación y contexto. Ante todo, se observan que pueden ser diferentes derechos y pretensiones a la hora de solicitar una calificación y, por tanto, el dictamen. Legalmente, en Colombia, a aquellas personas que obtienen una pérdida de capacidad ya sea laboral u ocupacional igual o mayor a 50\% se les considera en estado de invalidez. ${ }^{18}$

\footnotetext{
${ }^{17}$ Decreto 1507 del 2014 por el cual se expide el Manual Único para la Calificación de la Pérdida de la Capacidad Laboral y Ocupacional.

${ }^{18}$ La invalidez definida por el artículo 3 Decreto 1507 de 2014, el artículo 9 Ley 776 de 2002 y el artículo 38 de la Ley 100 de 1993 como la pérdida de la capacidad laboral u ocupacional igual o superior a cincuenta por ciento.
} 
Tabla 4. Distribución de las deficiencias por capítulos del título primero

\begin{tabular}{|l|l|}
\hline $\begin{array}{l}\text { Capítulo } 1 \text { por alteraciones debidas } \\
\text { a neoplasias o cáncer. }\end{array}$ & $\begin{array}{l}\text { Capitulo } 2 \text { por alteraciones } \\
\text { del sistema cardiovascular. }\end{array}$ \\
\hline $\begin{array}{l}\text { Capitulo } 3 \text { por trastornos } \\
\text { del sistema respiratorio. }\end{array}$ & Capítulo 4 del sistema digestivo. \\
\hline $\begin{array}{l}\text { Capitulo } 5 \text { del sistema urinario } \\
\text { y reproductor. }\end{array}$ & $\begin{array}{l}\text { Capítulo } 6 \text { por trastornos de la piel, } \\
\text { faneras y daño estético. }\end{array}$ \\
\hline $\begin{array}{l}\text { Capitulo } 7 \text { por alteraciones } \\
\text { del sistema hematopoyético. }\end{array}$ & $\begin{array}{l}\text { Capítulo } 8 \text { por alteraciones } \\
\text { del sistema endocrino. }\end{array}$ \\
\hline $\begin{array}{l}\text { Capitulo } 9 \text { por alteraciones } \\
\text { del sistema auditivo y vestibular. }\end{array}$ & $\begin{array}{l}\text { Capitulo } 10 \text { por alteraciones del olfato, del } \\
\text { gusto, de la voz, del habla y de las vías } \\
\text { aéreas. }\end{array}$ \\
\hline $\begin{array}{l}\text { Capitulo } 11 \text { por alteraciones } \\
\text { del sistema visual. }\end{array}$ & $\begin{array}{l}\text { Capitulo } 12 \text { Deficiencias del sistema } \\
\text { nervioso central y periférico. }\end{array}$ \\
\hline $\begin{array}{l}\text { Capitulo } 13 \text { Deficiencias por trastornos } \\
\text { mentales y del comportamiento. }\end{array}$ & $\begin{array}{l}\text { Capitulo } 14 \text { por alteración de las } \\
\text { extremidades superiores e inferiores. }\end{array}$ \\
\hline $\begin{array}{l}\text { Capitulo } 15 \text { por alteraciones } \\
\text { de la columna vertebral y la pelvis. }\end{array}$ & \\
\hline
\end{tabular}

Nota: Tabla adaptada por Suarez (2018) del anexo del Decreto 1507 de 2015.

\section{c) Enfoque metodológico}

Esta investigación se basó en un enfoque mixto. De acuerdo con Hernández, Fernández y Baptista, ${ }^{19}$ se refiere a un conjunto de procesos sistemáticos, empíricos y críticos donde se aplicaron aspectos relevantes de las perspectivas cualitativas y cuantitativas aplicadas a un objeto de estudio. Para lo anterior, el tipo de muestreo seleccionado fue no probabilístico o dirigido por conveniencia, o bien "guiadas por uno o varios propósitos".

Si bien el interés principal fue lograr la participación de por lo menos un médico por cada una de las 18 juntas existentes, como en todo proceso de investigación, hubo algunas limitaciones. En este caso, estuvieron dadas por el tamaño de la muestra, unidades de análisis y respuesta de participación por parte de los calificadores médicos.

La categorización requerida partió del planteamiento del problema, objetivo general y específicos, así como diferentes aspectos teóricos, no solo para

\footnotetext{
${ }^{19}$ Hernández Sampieri, Roberto, Fernández, Carlos y Baptista, Pilar, Metodologías de la Investigación, México, Mc Graw Hill, 2014 , p. 534.
} 
la justificación, sino también para el marco de antecedentes y referencial. La categorización expuesta respondió a las siguientes preguntas de investigación:

¿Qué dicen los planes de estudio de los programas de especialización de Seguridad y Salud en el Trabajo y la Medicina de Trabajo respecto a las competencias profesionales de los médicos para la valoración de las deficiencias, en el marco de la calificación de la pérdida de la capacidad laboral y ocupacional? ¿Cuál es la percepción de un grupo de médicos calificadores cuestionados respecto a las necesidades o requerimientos de formación para poder actuar como peritos en la aplicación del título primero del Manual de Calificación de Pérdida de Capacidad Laboral y Ocupacional?

Los instrumentos o apéndices de recolección de la presente investigación fueron diseñados de acuerdo con el objeto del presente estudio. Estos permitieron explorar tanto aspectos cualitativos como cuantitativos para caracterizar a la población. Asimismo, dieron pie a estrategias técnicas en el acercamiento, en un análisis objetivo de la praxis médica con la formación posgradual relacionada con la calificación de la pérdida de la capacidad laboral y ocupacional. Todo ello, tuvo como base la estructura del decreto, con énfasis en el título primero, valoración de las deficiencias, de competencia exclusivamente médica. Para el desarrollo del presente estudio fue necesario llevar a cabo una serie de fases, que incorporaron perspectivas cualitativas y cuantitativas, como se expone en el gráfico:

Gráfico 1. Fases del proceso investigativo

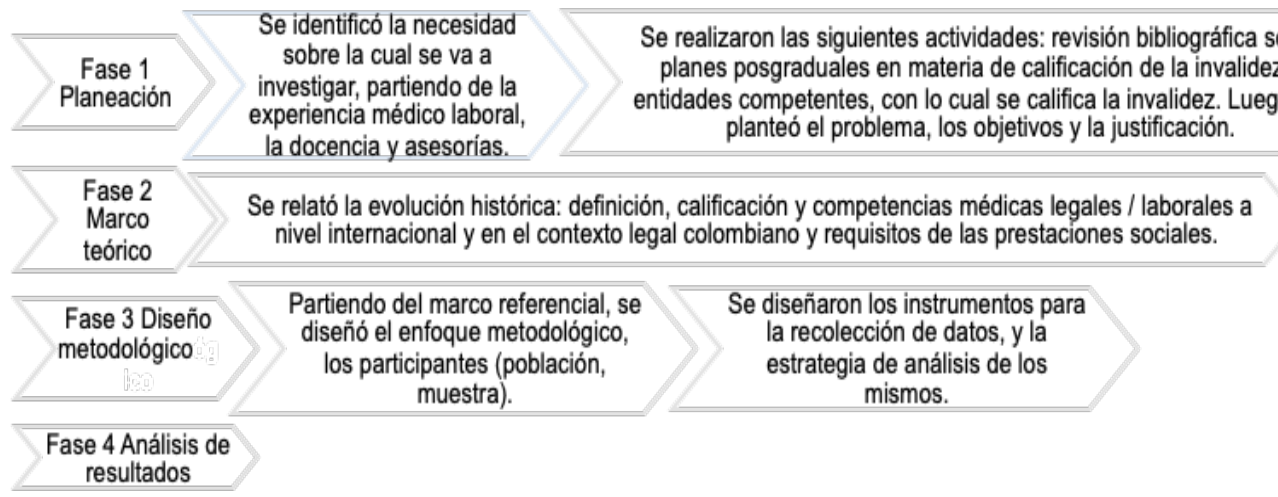

Nota: Elaborada por Suarez (2018). 


\section{Conclusiones}

Como lo exponen Hernández, Fernández y Baptista, ${ }^{20}$ los métodos mixtos permitieron el análisis combinado, el procesamiento individual de los datos cuantitativos (estadístico descriptivo e inferencial), así como de los datos cualitativos (codificación y evaluación temática). Las técnicas y modelos partieron de un análisis juicioso con el planteamiento del problema, el tipo de diseño y estrategias elegidas para los procedimientos, así como el análisis respectivo.

La relevancia de las siguientes conclusiones radica en que proyectan otros escenarios de la calificación. De tal modo, son de gran importancia para todos los profesionales médicos que hoy hacen parte de los equipos de calificación de primera oportunidad, entendida esta como la calificación realizada por las entidades administradores de la Seguridad Social Integral en Colombia (Administradoras de Riesgos Laborales, Salud y Pensiones), así como para terceros que en una dinámica de mercado competitivo pueden estar sacrificando calidad por bajos costos en el recurso humano empleado.

Respecto a la primera pregunta de investigación, si bien es cierto que uno de los requisitos legales para hacer parte de las Juntas de Calificación de Invalidez es tener especialización en Salud Ocupacional o en Medicina del Trabajo, resulta también cierto que la praxis médica de los miembros integrantes de estas juntas obliga a contar con el conocimiento del marco legal. Así, debe ser de su conocimiento el contenido y la interpretación de las normas técnicas como el Decreto 1507 de 2014, ${ }^{21}$ en especial en lo referente al título primero, inherente a las deficiencias. Sin embargo, también resulta necesario tener presente que el médico no califica solo a población trabajadora; califica a personas de cualquier edad y, por tanto, a seres diversos desde su misma funcionalidad y contextos.

Si bien se requiere la formación médica posgradual en Salud Ocupacional o en Medicina del Trabajo para poder ser miembro de las juntas de calificación u otro escenario, los planes de estudio revisados evidenciaron que solo la mitad de ellos ofrece algún contenido relacionado con el tema de la calificación de la pérdida de la capacidad laboral u ocupacional. De manera preocupante, la mitad de los programas no cuentan con una asignatura de medicina laboral, baremología o calificación, ni sobre el marco legal al respecto.

\footnotetext{
${ }^{20}$ Hernández Sampieri, Roberto, Fernández, Carlos y Baptista, Pillar, Metodologías de la Investigación, México, Mc Graw Hill, 2014, p. 574.

${ }^{21}$ Decreto 1507 de 2014: Por el cual se expide el Manual Único para la Calificación de la Pérdida de la Capacidad Laboral y Ocupacional.
} 
Queda la interrogante: si los planes de estudio no ofrecen estos mínimos, ¿cómo se puede llegar a la comprensión práctica, normativa y procedimental respecto a aspectos clave de la calificación? Ejemplo de estos aspectos son la incapacidad permanente parcial, estado de invalidez, mejoría médica máxima (ммм), carga de adherencia al tratamiento, pruebas para calificar, entre otros criterios, procedimientos y metodologías propias del título primero, contemplado en la norma.

En relación con lo anterior, los contenidos de los planes de estudio revisados se centran en aspectos de la promoción, prevención, mantenimiento y recuperación de la salud de los trabajadores, así como en la generación de espacios y prácticas de trabajo en seguridad, higiene y ambientes saludables de trabajo. Así, los planes de estudio no contemplan contenidos conceptuales, transversales y relacionados con la calificación de la pérdida de la capacidad laboral y ocupacional para diferentes poblaciones.

Desde este tipo de investigación, resulta incierto si se desarrollan los contenidos esperados en profundidad. Partiendo de la norma, es difícil definir qué aspectos deberían incluir los procesos en todo el contexto del Sistema de Seguridad Social Integral, puesto que los planes revisados no describen los objetivos, contenidos, temáticas, procedimientos y roles. A nivel prospectivo sería valioso llegar a desarrollar una investigación basada en el análisis de los currículos.

Respecto a la segunda pregunta, centrada en la valoración de lo expuesto por los médicos calificadores integrantes de juntas de calificación que voluntariamente accedieron a responder el instrumento generado, se halló que 100\% cuenta con el requisito mínimo exigido por la norma: estudios posgraduales en salud ocupacional, medicina del trabajo. Asimismo, es su totalidad expresan la necesidad que han tenido de adelantar cursos cortos y diplomados, con intensidades de hasta de 120 horas, respecto a la norma, baremo o herramienta actual de calificación. Esto significa la aproximación a nuevos saberes; no obstante, no garantizan la incorporación de competencias prácticas y cambios de paradigmas frente a los cambios en el conocimiento.

Lo expuesto anteriormente lleva a tener en cuenta las palabras del doctor Rodríguez de Castro:22 "si queremos desarrollar una nueva Medicina, es necesario que competencias que en estos momentos no tienen mucha visibilidad se hagan más evidentes en los planes de formación”.

\footnotetext{
${ }^{22}$ Escuela de Salud Pública, "Las competencias transversales del médico, vitales para atender con calidad al paciente", Mahón (Menorca). Disponible en: http://www.medicosypacientes.com/articulo/las-competencias-transversalesdel-medico-vitales-para-atender-con-calidad-al-paciente
} 
Frente a esto, los encuestados expresaron la falta de formación en aspectos generales, la legislación, y el contenido técnico y metodológico de todos los manuales o baremos de calificación. Esto es relevante sobre todo si se tiene en cuenta el contexto legal, que exige la aplicación de las normas anteriores, por las cuales se consolidaron los derechos adquiridos, como son las pensiones por incapacidad permanente parcial, total y las diferentes clases de pensiones de invalidez o de vejez anticipadas, así como por ser personas inválidas o presentar deficiencias iguales o superiores a 50\%.

Un médico calificador no puede conocer solo el decreto actual; debe conocer los anteriores y las normas aplicables a otros regímenes de excepción. Por lo anterior, es preciso reflexionar en torno a la necesidad de proponer a las entidades gubernamentales -entre otras, al Ministerio de Educación Nacional- que se generen normas de obligatorio cumplimiento para indicar a las entidades o instituciones universitarias que ofrecen programas posgraduales en Salud Ocupacional, publicar un mínimo sobre el contenido de los planes de estudio. Estos tendrían que señalar las asignaturas, los objetivos y su intensidad para que, de esa forma, el profesional interesado conozca de primera mano a qué se va a enfrentar. Esto significa ir más allá de presentar solo títulos de asignaturas o cursos.

Muchos profesionales en Colombia acceden a una oferta sesgada o desarrollada desde la perspectiva de salud ocupacional (ahora Seguridad y Salud en el Trabajo) ante la necesidad de contar con un título que les permita formar parte de los equipos calificadores. No obstante, esto dista de lo que implica realmente calificar tomando en cuenta las poblaciones y los enfoques actuales a nivel diferencial, territorial y de derechos, que parecieran ser más estudiados desde otras disciplinas. Por tanto, a la hora de llegar al mundo práctico y ejercicio profesional, diferentes frustraciones y prácticas no asertivas basadas en el desconocimiento o imaginarios terminan por afectar a las personas sujetos de calificación y a la calidad de los dictámenes esperados.

Hoy en día, la calificación en Colombia se enfrenta a diferentes barreras y lamentables situaciones que afectan el actuar ético y moral. Perspectivas de trabajo en equipo, de roles y saberes interdisciplinares siguen siendo arraigadas en imaginarios que no necesariamente van a la vanguardia del conocimiento histórico y actual.

Es importante transitar de lo exclusivamente médico a lo interdisciplinar a la hora de valorar y calificar a una persona. Por ello, surgen nuevas posibilidades para generar ofertas académicas a nivel de especialización, maestrías o doctorados, que profundicen en una experticia técnica como es la calificación de la pérdida de la capacidad laboral y ocupacional en el Sistema Integral de Seguridad Social. 
Es preciso reconocer que el acto de calificar exige romper paradigmas vigentes aún en Colombia, en donde se considera que la calificación es exclusivamente médica, contrario a lo señalado en las normas vigentes y a lo planteado desde lo académico.

\section{Referencias}

DAo, Alberto, El conocimiento y las competencias en las organizaciones del siglo XXI, Venezuela, Epsilon Libros - Universidad Metropolitana, 2002.

"Decreto 3170 de 1964: Por el cual se aprueba el reglamento general del seguro social obligatorio de accidentes de trabajo y enfermedades profesionales”, 21 de diciembre, 1964.

DíAz, Martha, "Reseña de Diez nuevas competencias para enseñar de Philippe Perrenoud”, Tiempo de Educar, vol. 9, núm. 17. Disponible en: http://ucsj.redalyc. org/articulo.oa?id=31111439008

Escuela de Salud Pública, "Las competencias transversales del médico, vitales para atender con calidad al paciente”, Mahón (Menorca). Disponible en: http://www. medicosypacientes.com/articulo/las-competencias-transversales-del-medicovitales-para-atender-con-calidad-al-paciente

Hernández Sampieri, Roberto, Fernández, Carlos y Baptista, Pilar, "Metodologías de la Investigación”, México, Mc Graw Hill, 2014.

KvitKo, Luis, “Antecedentes Históricos de la Valoración del Daño Corporal y Baremos”, Asociación Costarricense de Medicina Legal, vol. 32, núm. 81. Disponible en: http://www.scielo.sa.cr/pdf/mlcr/v32n1/art08v32n1.pdf

"Ley 860 del 2003: Por la cual se reforman algunas disposiciones del Sistema General de Pensiones" previsto en la Ley 100 de 1993 y se dictan otras disposiciones. Diario oficial No 45.415

"Ley 90 de 1946: Por la cual se establece el seguro social obligatorio y se crea el Instituto Colombiano de Seguros Sociales”, Diario Oficial, núm. 26322.

"Ley 776 del 2002, Por la cual se dictan normas sobre la organización, administración y prestaciones del Sistema General de Riesgos Profesionales”, Diario oficial, núm. 45037.

Moreno, Marisol, Valoración de la pérdida de la capacidad laboral y ocupacional en Colombia: antecedentes, realidades y perspectivas de actualización, Bogotá, Universidad Nacional de Colombia, 2011.

Organización Mundial de la Salud, Clasificación Internacional del Funcionamiento, la Discapacidad y la Salud, Ginebra, Organización Mundial de la Salud, 2001.

Sosa, Ángela y CAPote, SiLVia, "Modelo tradicional versus enfoque por competencias", Uruguay Educa. Disponible en: http://www.uruguayeduca.edu.uy/sites/default/ files/2017-08/MODELO_TRADICIONAL_vs_ENFOQUE_por_COMPETENCIAS.pdf 